\title{
Immunohistochemical Detection of Viral Etiopathogenesis in Lambs and Goat Kids with Neonatal Diarrhea
}

\author{
Ozlem Ozmen', Mehmet Haligur², Ahmet Aydogan² \& Necdet Demir ${ }^{3}$
}

\begin{abstract}
Background: Neonatal enteritis is an important disease that causes deaths of animals before 3 weeks of age, and results in significant economic losses. Viral agents can predispose the young animals to secondary infections in the gastrointestinal tract, especially in lambs and goat kids younger than 21 days. Although the neonatal diarrhea is common in calves, there is still little knowledge about pathology, pathogenesis and immunohistochemical localization of viral agents that cause neonatal enteritis in lambs and goat kids. In this study, we carried out investigations with the aim of detecting adenovirus, rotavirus, coronavirus and herpes virus in the guts of goat kids and lambs with viral enteritis.

Materials, Methods \& Results: Adenovirus, rotavirus, coronavirus and herpes virus antisera were applied to paraffinembedded intestinal tissue from neonatal lambs and kids that had died from enteritis. In addition, viral agents in the gut cells were detected and evaluated by electron microscopy. The study material consisted of 15 lambs and 15 goat kids that were presented for diagnosis. Viral agents were detected by immunohistochemically in 20 out of 30 animals. Rotavirus was diagnosed in 10 animals, adenovirus in five, herpes virus in three and coronavirus in two animals; and these results were supported by the electron microscopy. This study showed that viral agents play an important role in neonatal enteritis in lambs and kids.

Discussion: Bacteria, viruses and protozoa may have a role in the etiology of neonatal enteritis and identifying the etiological agents is not always possible without laboratory studies. In addition, the immune system of the animal and environmental factors are important factors for to occurrence of the disease. The ages of the animals in present study varied between 1 and 21 days, but susceptibility to infection was most commonly observed between the ages of 1 and 12 days, and the infected animals immediately died after the appearance of clinical symptoms during this period. The most common clinical symptoms were fever, depression, dehydration, tenesmus, abdominal swelling and loss of appetite. Histopathological examinations revealed mild to severe lesions in the gut samples including hyperemia of vessels, submucosal edema, desquamation, erosion and ulcers of the epithelial layer of the gut, and inflammatory cell infiltration in the submucosa. Enlargement of mucus-secreting cells was most commonly observed. These results are common in neonatal enteritis and an agreement with previous studies and classical knowledge about clinical and pathological findings in this study. The agents of neonatal diarrhea most often isolated in calves are rotavirus, coronavirus, Cryptosporidium and enterotoxigenic Escherichia coli. A diagnosis of viral agents can be made by immunohistochemical examination of tissue fixed in formalin. This study revealed that the most common viral agents in lambs and goat kids neonatal diarrhea was rotavirus. In this study, protozoal and bacterial contamination was also observed. Our current study also showed that coronavirus is not an important etiological factor, compared with other viral causes of diarrhea in small ruminants. However, according to a previous study by our group, its incidence has increased and may be an important viral agent in neonatal enteritis in the future. This study showed that viral etiology was very important to occurring of neonatal diarrhea, practitioners must be taken into consideration when meet this problem. One of the most important prophylactic measurements may be vaccination pregnant sheep or goat for passive immunity of offspring.
\end{abstract}

Keywords: enteritis, lambs, goat kids, adenovirus, rotavirus, coronavirus, herpes virus.

DOI: $10.22456 / 1679-9216.83864$ 


\section{INTRODUCTION}

Neonatal enteritis in goat kids and lambs 21 days of age, or younger, is one of the most common diseases causing high morbidity and mortality in these animals $[2,10,14]$. The impaired growth of neonatal goat kids and lambs afflicted with enteritis, the expenditures involved in treating these animals, and their increased mortality all have costly economic losses $[2,10]$. Rotaviruses, adenoviruses, herpes viruses, and coronaviruses appear to be the most common viruses that cause enteritis in humans and animals $[14,15]$.

Adenoviruses are important agents of enteritis in humans, cattle, pigs, horses, sheep, goats and dogs $[5,11,13]$. Different serotypes are found in different species, and they are commonly found worldwide [5,9]. Caprine herpes viruses have been isolated from kids in different countries. These viruses can infect sheep, cattle and goats, but severe infections only occur in goats. Goat kids die 1-4 days after clinical symptoms start. Lesions may occur throughout the gastrointestinal tract [5,9,21].

Coronaviruses may cause problems in calves, especially those between 4-30 days of age. In our previous study, we found coronavirus in the gut of one of the 30 newborn lambs and goat kids that died from neonatal enteritis [19].

There is, however, little known about the etiopathology and localization of viral agents of neonatal enteritis in lambs and kids. In this study, we used pathological examinations, immunohistochemistry and electron microscopy to examine gut samples from goat kids and lambs with neonatal enteritis with the aim of evaluating the roles of adenovirus, rotavirus, coronavirus and herpes virus in the etiopathology of the disease and localization of viral agents in intestines.

\section{MATERIALS AND METHODS}

\section{Samples}

In this study, 15 goat kids and 15 lambs that died of enteritis at the age of 21 days or younger were examined for rotavirus, adenovirus, herpes virus and coronavirus by immunohistochemical methods. All of the goat kids and lambs were obtained from farms near the Burdur, Turkey. All of the lambs and goat kids were from house reared family crowded farms and under lower hygienic conditions. Different ages group animals were keep together and in higher mortality cases the main problem was the porter and big animals. A total of
30 animals were in study group and ages of the animals were between 3 to 21 days but, 18 animals were under the 10-day of age. The guts of five neonatal goat kids and five neonatal lambs that died from other causes were used as controls. During necropsy, gut contents were also collected for parasitological examination.

\section{Histopathological analysis}

Gut and mesenteric lymph node samples were collected and fixed in $10 \%$ buffered formalin. After routine processing, tissues were embedded in paraffin and sectioned to 5 - $\mu \mathrm{m}$ thickness. Tissue sections were stained with Hematoxylin-eosin (HE) and examined microscopically. Morphometric analyses such as thickness of the gut wall, increase of the mucus secreting cells and size of the cells were examined. Morphometric evaluation was made by using the Database Manual Cell Sens Life Science Imaging Software System ${ }^{1}$.

\section{Immunohistochemical analysis}

Gut samples were then immunostained with adenovirus antiserum [adenovirus type 5 antibody (ab6982), 1/100 dilution $]^{2}$, rabbit polyclonal antibody to novel coronavirus (HCoV-EMC/2012) nucleocapsid protein (catalog number 100211-RP02; 1/100 dilution $^{3}$, rotavirus antibody, (rotavirus polyclonal antibody, catalog number MBS534886, 1/100 dilution $)^{4}$, and herpes virus antiserum (polyclonal rabbit antibody to herpes simplex virus type I (HSV 1); catalog number RP 018; $1 / 100$ dilution) $)^{5}$, according the manufacturer's instructions by using a routine streptavidin-biotin peroxidase technique. Expose mouse and rabbit specific HRP/DAB detection IHC kit (ab80436) ${ }^{2}$ was used as secondary antibody. All the slides were analyzed for immunopositivity for the viral agents. The five goat kids and five lambs' guts that died from other causes in the neonatal period were used as controls. Positive controls were used for each viral agent. Primary antibodies were not applied to the negative controls for immunohistochemistry.

\section{Electronmicroscopical analysis}

Gut samples were also processed for transmission electron microscopy (TEM). They were fixed in $2.2 \%$ glutaraldehyde and post-fixed in $1 \%$ osmium tetroxide $(\mathrm{OsO} 4)$ prepared in $0.1 \mathrm{M}$ phosphate buffer solution. The samples were dehydrated in a graded alcohol series and embedded in Araldite CY212. Ultrathin sections were taken from the plastic blocks, 
stained with uranyl acetate/lead citrate, and examined with a JEOL JEM 1220 model TEM.

\section{RESULTS}

The ages of the animals varied between 1 and 21 days, but susceptibility to infection was most commonly observed between the ages of 1 and 12 days, and the infected animals immediately died after the appearance of clinical symptoms during this period. According to the owner's anamnesis, the newborn goat kids and lambs that died at an age less than one week had presented either no clinical signs, or mild enteritis, whereas those newborns that died when they were more than 1-week old had continuous diarrhea for 1 or 2 days before dying. The most common clinical symptoms were fever, depression, dehydration, tenesmus, abdominal swelling and loss of appetite. Hypothermia commonly occurred before death. The diarrhea was characteristically watery, and with a yellowish-white hemorrhagic guts content. Mortality rates depended on management and breeding conditions and varied $25-50 \%$. Hyperemia in the mesenteric vessels, mesenteric lymphadenopathy, and gas and watery fluid were commonly observed in the small and large intestines during necropsy (Figure 1). Generally serous fluid was seen in the abdominal cavity, and abomasal bleeding was seen in most of the cases. Edema and hemorrhages were commonly observed in the gut mucosae. Giardia intestinalis was found in three cases upon fresh feces examination, and Cryptosporidium parvum was found in eight cases after the gut contents were stained with carbol fuchsin. In some cases, multiple types of bacteria were observed in the contents of the gut.

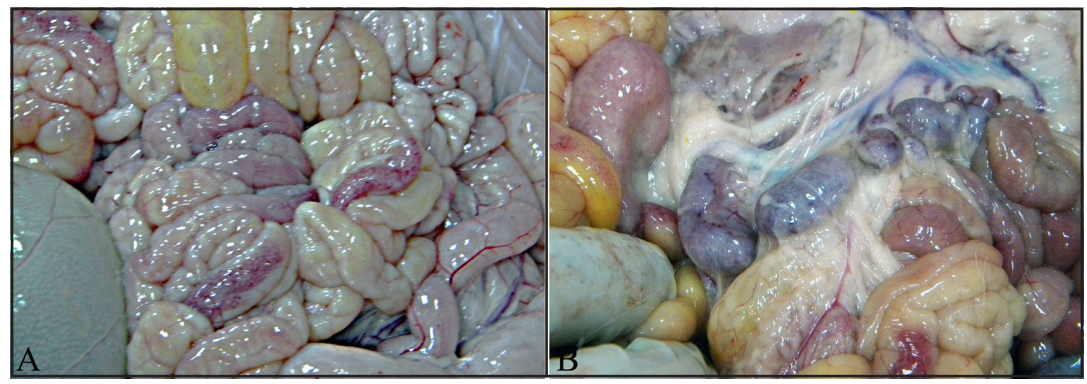

Figure 1. A- Gross appearance at necropsy of the guts of a kid with neonatal enteritis. B- enlargement of the mesenteric lymph nodes.

Histopathological examinations revealed mild to severe pathological changes in the gut samples. The most common findings were hyperemia of vessels, submucosal edema, desquamation, erosion and ulcers of the epithelial layer of the gut, and inflammatory cell infiltration in the submucosa. Enlargement of mucus-secreting cells was observed in 28 of 30 gut samples. In severe cases, total necrosis of the mucosa was observed. Desquamation and infiltration of the propria mucosa were noted in all cases (Figure 2). Only mild pathological lesions were observed in those animals that died suddenly and were less than 1 week old, and inflammation was generally due to neutrophil leucocytes. The intestinal villi of goat kids and lambs more than 10 days old were commonly observed to be shortened and fused. Enlarged lacteals, swollen goblet cells and regenerative cells were observed in mild cases. No inclusion bodies were observed in this study. The pathological findings diagnosed in lambs were similar to those diagnosed in goat kids. It was not possible to diagnose a viral etiology with only a histopathological examination. In eight cases a viral etiology was combined with Cryptosporidium parvum infection and in five cases numerous bacterial colonies were observed. Infection with a combination of protozoa and bacteria increased the severity of the lesions. Lymphadenopathy and neutrophil infiltrations were observed in the mesenterial lymph nodes.

Immunohistochemical examinations revealed rotavirus in 10 animals $(33.3 \%)$, adenovirus in five animals $(16.6 \%)$, herpes virus in three animals (10\%) and coronavirus in two animals $(6.6 \%)$.

For the rotavirus-positive cases, differentiation from columnar epithelium to cuboidal epithelium and villous fusion were common findings. The immunopositive cells were localized in the small intestines, especially in the ileum, and there was an immunopositive reaction of the inflammatory cells. Histopathological examinations of the rotavirus-positive cases revealed focal areas of vacuolar degeneration of the epithelial lining, mainly at the tips of the villi, in the jejunum. Cytoplasmic vacuolization was observed in the ileum. Lesions were generally localized in the small intestines and slight inflammatory reactions occurred in the propria mucosa (Figure 3A). 


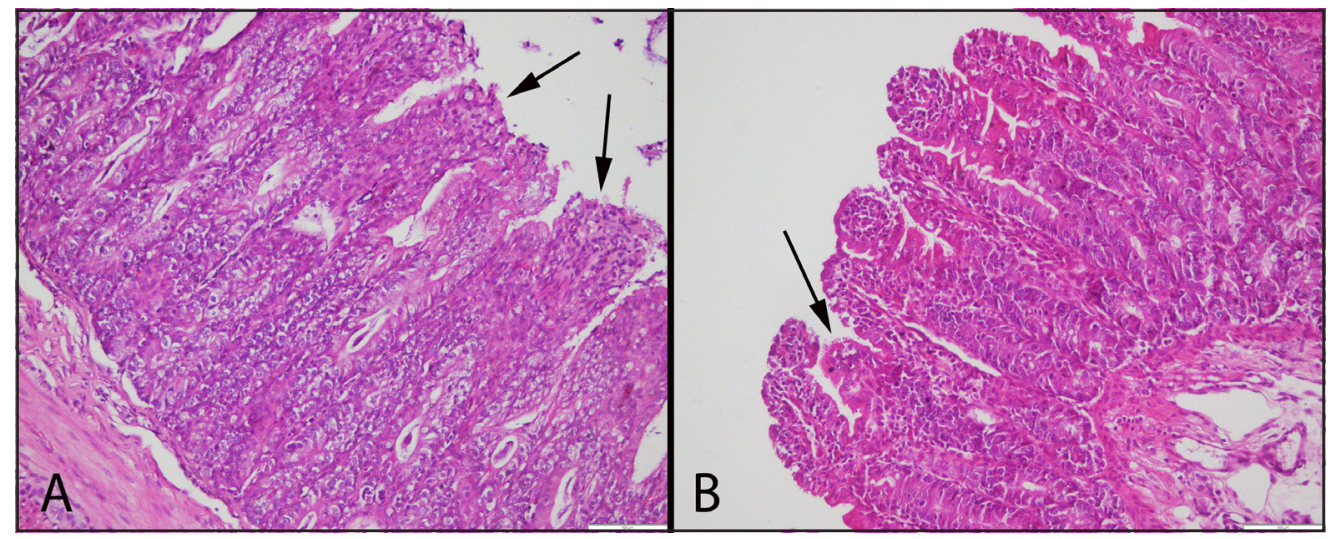

Figure 2. Histopathological appearance of the gut of a lamb with neonatal enteritis. A- Desquamation of the villus (arrows). B- Fusion of the villus (arrow) [HE. Bar= $100 \mu \mathrm{m}]$.

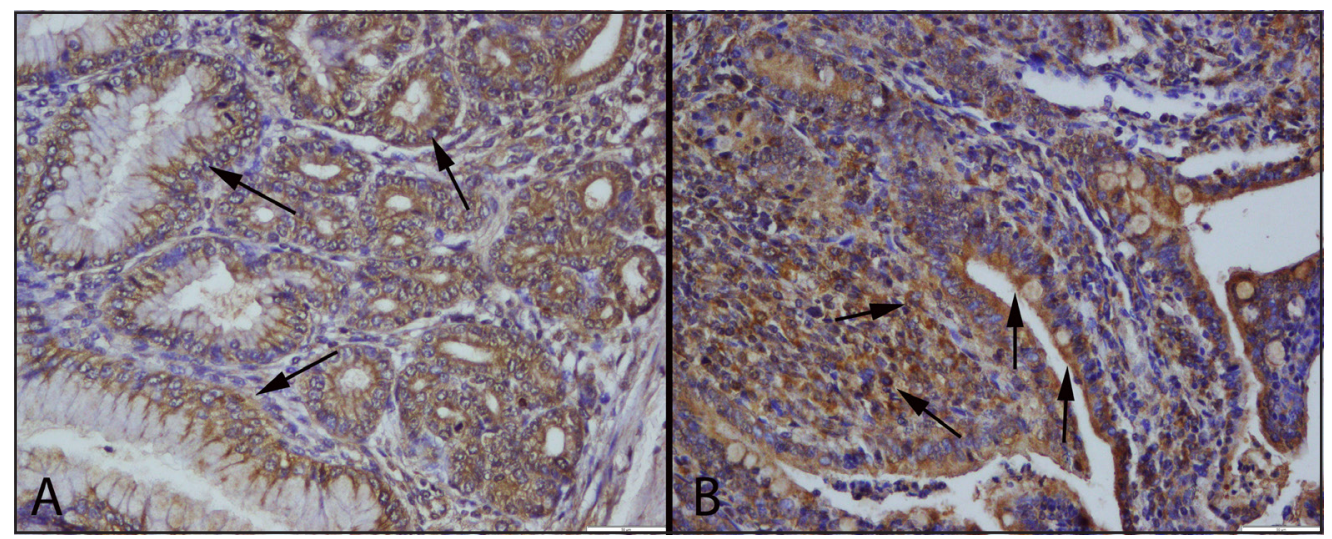

Figure 3. A- Rotavirus immunoreaction in the enterocytes (arrows) [Bar= $100 \mu \mathrm{m}]$. B- Adenovirus-positive cells in the guts detected using the streptavidin-biotin-peroxidase method [Bar= $50 \mu \mathrm{m}]$.

Adenovirus-positive immunohistochemical reactions were observed in three goat kids and two lambs. Adenovirus was detected in enterocytes, crypt epithelial cells and inflammatory cells. Adenovirus-positive reactions were localized to the small and large intestines. Desquamation of adenovirus-positive cells and severe inflammatory reactions of the propria mucosa were common findings of the histopathological examination. Neither inclusion bodies nor villous fusion were observed (Figure 3B)

Immunopositive reactions for herpes virus were observed throughout the gastrointestinal tract, but were severe in the cecum. These reactions were evident in enterocytes and lymphoid cells, and were marked in goblet cells. Erosive and ulcerative lesions were observed in both the small and large intestines in histopathological examinations of herpes virus-positive cases. Neither inclusion bodies nor villous fusion was observed (Figure 4A).

Coronavirus-positive immunoreactions were observed both in the small and large intestines but were mainly localized to the jejunum. These reactions were evident in enterocytes, crypt epithelial cells and macrophages in the propria mucosa. This agent generally localized in the api- cal portion of the cells. Desquamation and erosion of the enterocytes and degeneration of goblet cells were observed during the histopathological examination (Figure 4B). There was no viral immunopositivity observed in lymph nodes.

Transmission electron microscopy (TEM) revealed viral particles in the enterocytes of immunopositive cases (Figures $5 \& 6$ ). Degenerated organelles and numerous granules were observed in virus-infected cells. In rotavirus positive cases, especially in the jejunum and ileum, some necrotic villus epithelial cells, and a small number of virus particles in the cells, were observed. The viral particles in the gut epithelial cells were located in the basal cytoplasm of the cell. Adenoviruses were recognized as particles in the cytoplasm of enterocytes and caused degeneration on cytoplasmic organelles. TEM examination of herpes virus positive cases revealed viral particles, such as capsids or enveloped virions, in the cytoplasm of the enterocytes. The coronavirus positive cases revealed morphological features of cytoplasmic structures. Small numbers of virions were seen at various stages of budding from the plasma membrane or free viral particles in the cytoplasm. 


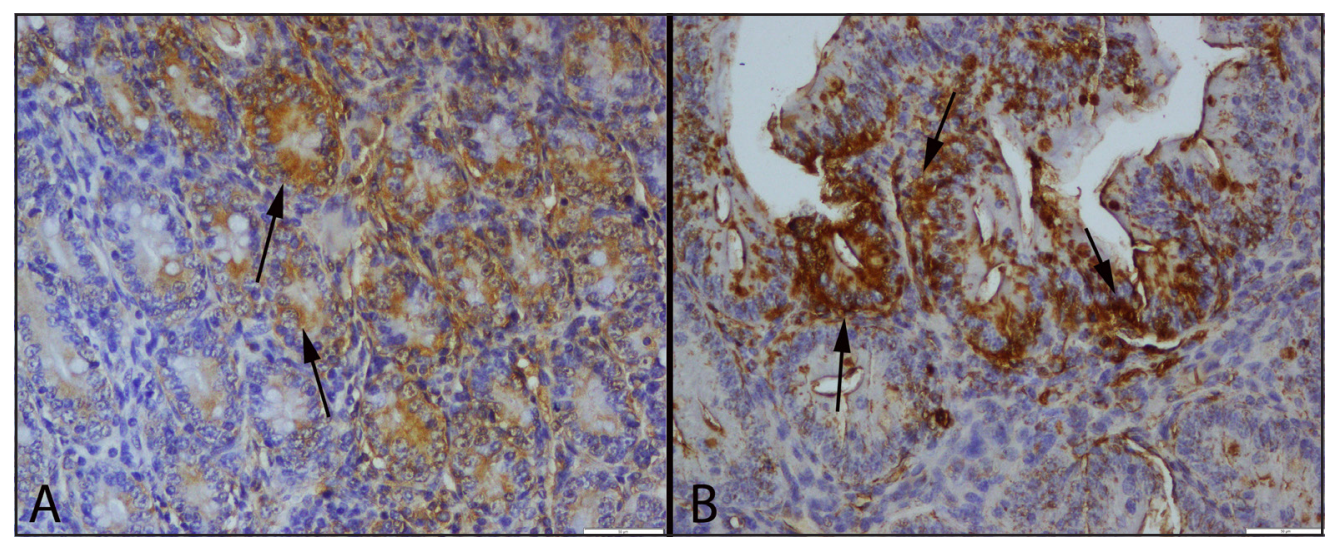

Figure 4. A- Herpesvirus-positive immunoreaction in gut cells (arrows). B- Coronavirus immunoreaction in the enterocytes (arrows), detected using the streptavidin-biotin-peroxidase method [Bar=50 $\mu \mathrm{m}$ ].

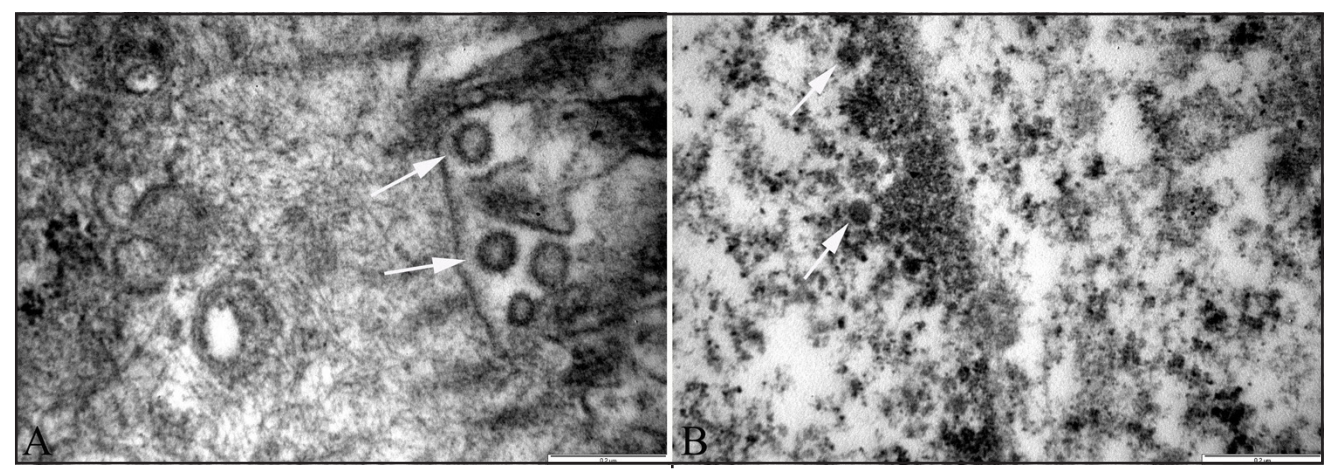

Figure 5. A- Adenovirus-positive particles (arrows). B- herpesvirus-positive particles (arrows) observed using transmission electron microscopy. [Bars $=0.2 \mu \mathrm{m}]$.

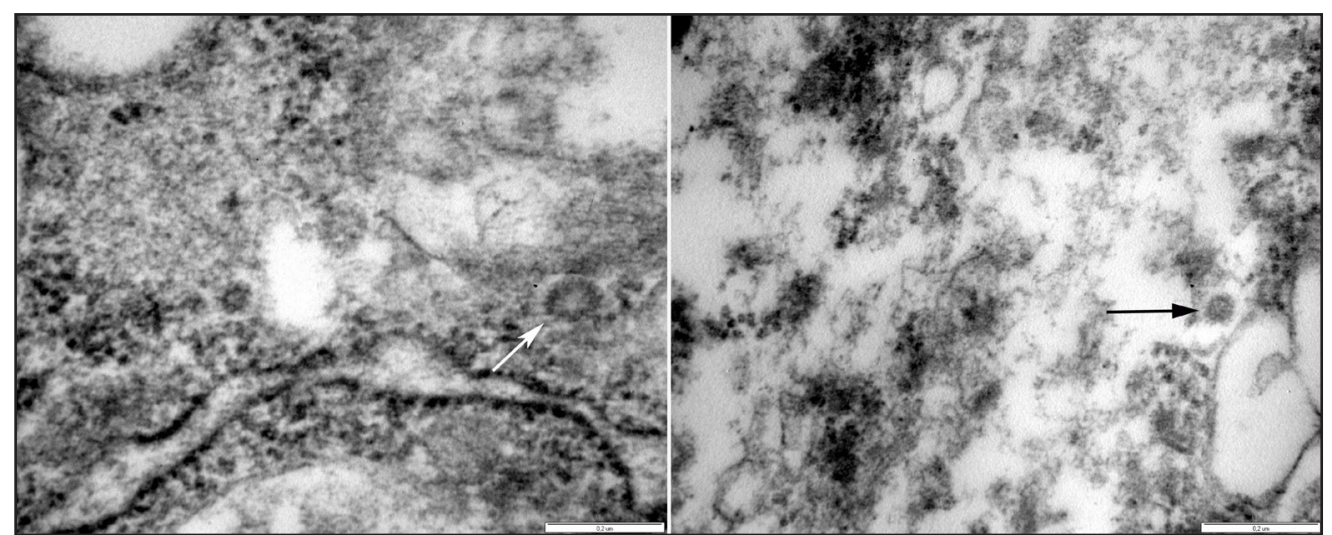

Figure 6. A- Coronavirus-positive particle (arrow). B- rotavirus-positive particle (arrow) observed using transmission electron microscopy. [Bars $=0.2 \mu \mathrm{m}]$.

\section{DISCUSSION}

Neonatal enteritis is a significant disease that causes deaths of animals 21 days of age or younger, and results in significant economic losses. There is still no effective treatment available to control enteritis in newborn animals. Identifying the etiological agents is not always possible, and field and laboratory studies have shown that there is no single agent in the etiol- ogy of neonatal enteritis. Enteropathogenic bacteria, viruses and protozoa, as well as the immune system of the animal and environmental factors, all play roles in the disease. It is also not always possible to clinically distinguish between the various etiological factors of neonatal diarrhea $[3,4]$. The agents of neonatal diarrhea most often isolated in calves are rotavirus, coronavirus, cryptosporidium and enterotoxigenic 
E. coli. $[3,8,16,17,21]$. This study revealed that the most common viral agents in lamb and goat kid neonatal diarrhea was rotavirus. In this study, protozoal and bacterial contamination was also observed.

Acute illness due to neonatal enteritis may be characterized by progressive dehydration, and death sometimes occurs in a period as short as $12 \mathrm{~h}$. In the subacute form of the disease, diarrhea may continue for several days, which can result in malnutrition and weight loss. The principles of the pathophysiology and treatment of enteritis in lambs and goat kids are the same as those for calves [12]. Altogether, however, relatively few immunohistochemical investigations have been reported regarding neonatal diarrhea in these animals. This study showed that goat kids and lambs were more susceptible for neonatal enteritis than calves.

Thus, our aim was to conduct a survey of the prevalence of selected viral etiological factors of enteritis in neonatal goat kids and lambs, and we found that there was an important viral etiology in these animals. We investigated the prevalence of adenovirus, coronavirus, herpes virus and rotavirus in lambs and goat kids, and described the specific clinical and pathological manifestations of each of these infections. We gained valuable information on the etiopathological factors of neonatal enteritis in these animals. Rotaviruses and adenoviruses were found to be the most common etiological viruses causing diarrhea in the neonatal lambs and goat kids we studied.

The occurrence of enteritis in the neonatal period is due to various enteropathogens and the frequency and importance of these enteropathogens varies geographically. However, the most common infections in many parts of the world involve Escherichia coli, rotavirus, coronavirus and Cryptosporidium parvum. Neonatal diarrhea is frequently caused by more than one of these pathogens, and severe outbreaks usually have multiple causes. Identification of sources of outbreaks is very important for effective treatment [6]. We observed similar findings regarding the development of the diarrhea. We found that neonatal care and feeding conditions are important factors affecting the severity of the disease. In the herds that had poor sanitary and hygienic measures and care in place, high mortality was observed, and in these flocks a combination of Cryptosporidium and Giardia was noted.

Viral pathogens are very important in the causation of neonatal diarrhea. Among these, rotavirus is the most common viral cause of diarrhea in calves and lambs. Rotaviruses are classified into groups " $A$ " and "B", and group A is the group that most commonly causes infection. Several different clinically significant virulent serotypes have been isolated from cases of rotaviral enteritis. Rotavirus functions by multiplying in the cell, then breaking down the cell and infecting adjacent cells. The shedding of the virus in groups of cells causes new cells to become infected. Loss of intestinal absorptive cells, i.e., enterocytes, especially in the small intestine, leads to a decrease of mature cells. Enterocytes are lost when infected by virulent rotavirus strains at a rate that exceeds the regenerative capacity of the intestinal epithelial crypt, and clinical effects result. There is a decrease in the intestinal absorptive surface and villus height, with a resulting decrease in the total activity of intestinal digestive enzymes $[12,15,20]$. Similar findings were observed in our study, in which cell loss was commonly observed in coronavirus-infected intestines. Such cell loss has been most frequently observed in rotavirus-infected cases.

Coronavirus diseases create problems at any age, but especially in young ruminant animals between four and 30 days old. Their lesions are located in the gut. Coronavirus has been observed to cause degradation of absorptive epithelial cells in the small intestine and to produce severe villous atrophy. Pathological lesions are common in the spiral colon, and they sometimes result in colitis that is clinically characterized by blood and mucus in the stool $[7,17]$. Similar pathological findings were observed in our current study and coronaviruses were detected in two of the animals.

As described in the introduction, coronaviral enteritis is clinically characterized by dehydration, weight loss, yellowish, viscous, watery, and at times, bloody, diarrhea, depression and anorexia. The course of the infection is more prolonged and the clinical symptoms are more extensive in coronaviral enteritis than in the rotavirus-induced disease, and diarrhea was observed to occur 2 to 4 days after the tissue was first damaged. The gross lesions produced by coronavirus enteritis are indistinguishable from those of rotavirus enteritis. The small and large intestines are swollen and filled with a moderate amount of yellowish content. Coronaviral enteritis has been microscopically observed to start in the proximal small intestine and spread to the villous epithelium of the colon. Immunofluorescence studies showed that viral antigen was present in all the cells surrounding 
the villi with the onset of diarrhea. After the onset of diarrhea, viral antigen is present in the crypt epithelium and remains there for 3-4 days. Within the first 48-96 $\mathrm{h}$ of the disease, the epithelial cells become necrotic and are transformed into immature cuboidal or squamous epithelium cells surrounded by markedly shortened villi. The superficial epithelium of the colon is replaced by immature cells. In the crypt, the lumen degenerates and necrotic cells are found. Focally hyperplastic crypts and numerous lymphocytes and plasma cells are observed in the part of the epithelium below the surface. Fibroblasts, histiocytes and infected endothelial cells are observed in reduced numbers in the lamina propria and focal necrosis is seen in these areas. Paracortical sinuses of the regional lymph nodes are filled with nuclear particles of lymphocytes, plasma cells and macrophages. The hemorrhagic form of coronavirus enterocolitis affects the colonic mucosa. Whether this is a very virulent strain of the disease, or whether other pathogens are involved to result in this hemorrhagic state is not known [4]. Our study showed a coronavirus positive reaction in two patients, and this was observed as granular structures within the enterocytes. We did not observe coronaviral immunopositivity in the lymph nodes.

A diagnosis of coronavirus can be made by immunohistochemical examination of tissue fixed in formalin [6]. Coronavirus is an important etiological factor in calf diarrhea but is less important in the neonatal diarrhea of lambs and goat kids $[1,7,8,16]$. Our current study also showed that coronavirus is not an important etiological factor, compared with other viral causes of diarrhea in small ruminants. However, according to a previous study by our group, its incidence has increased and may be an important viral agent in neonatal enteritis in the future.
There are many published studies that have aimed to identify the etiological factors of neonatal diarrhea in lambs and goat kids, and most of these studies have generally involved electron microscope examinations of fecal samples $[1,8,12,16,18]$. However, there are few immunohistochemical studies of etiological factors of neonatal enteritis. The pathological examinations reported here of adenovirus, coronavirus, herpesvirus and rotavirus in neonatal lambs and goat kids presenting diarrhea showed rotavirus to be the most common agent. The study also noted that the occurrence of neonatal enteritis is very much dependent on the hygienic measures taken.

\section{CONCLUSIONS}

In conclusion, this study showed that viral etiology was very important to occurring of neonatal diarrhea, practitioners must be taken into consideration when meet this problem. One of the most important prophylactic measurements may be vaccination pregnant small ruminants for passive immunity of goat kids and lambs.

\author{
MANUFACTURERS \\ ${ }^{1}$ Olympus Corporation. Tokyo, Japan. \\ ${ }^{2}$ Abcam. Cambridge, UK. \\ ${ }^{3}$ Sino Biological Inc. Beijing, China. \\ ${ }^{4}$ MyBioSource. San Diego, CA, USA. \\ ${ }^{5}$ Diagnostic BioSystems. Pleasanton, CA, USA.
}

Acknowledgements. This study was supported by Scientific Projects Commission of University of Mehmet Akif Ersoy (Project number: 0196-NAP-13).

Declaration of interest. The authors report no conflict interest. The authors alone are responsible for the content and writing of paper.

\section{REFERENCES}

1 Abou El Hassan D.G. 1996. Neonatal diarrhoea in lambs and goat kids. In: Proceedings of the Fourth Scientific Congress, Veterinary Medicine and Human Health (Cairo, Egypt). pp.371-380.

2 Andrés S., Jiménez A., Sánchez J., Alonso J.M., Gomeza L., Lopez F. \& Rey J. 2007. Evaluation of some etiological factors predisposing to diarrhoea in lambs in "La Serena" (Southwest Spain). Small Ruminant Research. 70(2-3): 272-275.

3 Blood D.C. \& Radostits O.M. 1989. Disease of the nervous system. In: Blood D.C. \& Radostits O.M. (Eds). Veterinary Medicine. 2nd edn. London: Bailliere and Tindall, pp.619-821.

4 Blood D.C. 1997. Pocket Companion to Veterinary Medicine. London: Bailliere Tindall, pp.458-459.

5 Brown C.C., Barker D.C. \& Barker I.K. 2007. Alimentary Systems. In: Maxie M.G. (Ed). Jubb, Kennedy and Palmer's Pathology of Domestic Animals. Philadelphia: Saunders Elsevier, pp.135-177. 
6 Daginakatte G.C., Chard-Bergstrom C., Andrews G.A. \& Kapil S. 1999. Production, characterisation, and uses of monoclonal antibodies against recombinant nucleoprotein of elk coronavirus. Clinical and Diagnostic Laboratory Immunolgy. 6(3): 341-344.

7 Durham P.J.K., Stevenson B.J. \& Farquharson B.C. 1979. Rotavirus and Coronavirus associated diarrhoea in domestic animals. New Zealand Veterinary Journal. 27(3): 30-32.

8 Eisa M.I. \& Mohamed A.A. 2004. Role of enteric pathogens in enteritis in lambs, goat kids and children and their zoonotic importance. Veterinary Medical Journal of Giza. 52(1): 41-59.

9 Gelberg H.B. 2001. Alimentary System. In: McGavin M.D., Carlton W.W. \& Zachary J.F. (Eds). Thomson's Special Veterinary Pathology. Saint Louis: Mosby, pp.48-50.

10 Gokce E. \& Erdogan H.M. 2009. An epidemiological study on neonatal lamb healthy. Kafkas University Veterinary Faculty Journal. 15(2): 225236.

11 Hervas J., Lopez S., De Lara F.C.M., Carrasco J.A.L. \& Gomez-Villamandos J.C. 1998. Adeno-virus infection in Spanish Ibex. Journal of Veterinary Diagnostic Investigation. 10(1): 97-100.

12 Kahn C.M. \& Line S. 2005. Merck Veterinary Manual. 9th edn. Wellington: Merck, 2700p.

13 Lehmkuhl H.D., Debey B.M. \& Cutlip R.C. 2001. A new serotype adenovirus isolated from a goat in the United States. Journal of Veterinary Diagnostic Investigation. 13(3): 195-200.

14 Martellaa V., Decaroa N. \& Buonavoglia C. 2015. Enteric viral infections in lambs or kids. Veterinary Microbiology. 181(1-2): 154-160.

15 Matthijnssens J., Potgieter C.A., Ciarlet M., Parreño V., Martella V., Bányai K., Garaicoechea L., Palombo E.A., Novo L., Zeller M., Arista S., Gerna G., Rahman M. \& Van Ranst M. 2009. Are human P[14] rotavirus strains the result of interspecies transmissions from sheep or other ungulates that belong to the mammalian order Artiodactyla? Journal of Virology. 83(7): 2917-2929.

16 Munoz M., Alvarez M., Lanza I. \& Carmenes P. 1996. Role of enteric pathogens in the aetiology of neonatal diarrhoea in lambs and goat kids in Spain. Epidemiology and Infection. 117(1): 203-211.

17 Naylor J.M. 1990. Diarrhoea in Neonatal Ruminants. In: Smith B.P. (Ed). Large Animal Internal Disease. Saint Louis: Mosby Publishing, pp.348-353.

18 Olson E.J., Haskell S.R.R., Frank R.K., Lehmkuhl H.D., Ann Hobbs L., Warg J.V., Landgraf J.G. \& Wunschmann A. 2004. Isolation of an adenovirus and an adeno-associated virus from goat kids with enteritis. Journal of Veterinary Diagnostic Investigation. 16(5): 461-464.

19 Ozmen O., Yukari B.A., Haligur M. \& Sahinduran S. 2006. Observations and immunohistochemical detection of coronavirus, Cryptosporidium parvum and Giardia intestinalis in neonatal diarrhoea in lambs and kids. Schweizer Archiv fur Tierheilkunde. 148(7): 357-364.

20 Papp H., Malik Y., Farkas S., Jakab F., Martella V. \& Banyai K. 2014. Rotavirus strains in neglected animal species, including lambs, goats and camelids. Virus Disease. 25(2): 215-222.

21 Steele A.D., Geyer A. \& Gerdes G.H. 2004. Rotavirus Infections. In: Coetzer J.A.W. \& Tustin R.C. (Eds). Infectious Diseases of Livestock. New York: Oxford University Press, pp.1256-1264. 